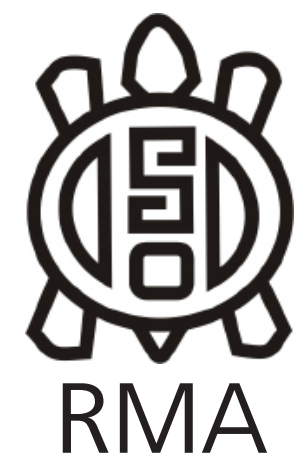

Dossier

\title{
Aproximaciones Traceológicas a la Cerámica del Primer Milenio d. C. de Quebrada de Los Corrales (El Infiernillo, Tucumán, Argentina)
}

\author{
Traceologic Approach to the First Millenium A.D Ceramics of Quebrada \\ de Los Corrales (El Infiernillo, Tucumán, Argentina)
}

Carlos Matías Gramajo Bühler*, Jaume García Rosselló**

*Instituto de Arqueología y Museo, Facultad de Ciencias Naturales e IML-UNT/Instituto Superior de Estudios Sociales (CONICET-UNT)/Fundación Miguel Lillo. E-mail: cmatiasgb@gmail.com

** Departamento de Cències Històriques i teoría de les Arts. Grupo de investigación arqueoUIB. Universitat de les Illes Balears. E-mail: Jaume.garcia@uib.es

\begin{abstract}
Resumen
Quebrada de Los Corrales corresponde a una localidad arqueológica rica en evidencias que dan cuenta de diversas ocupaciones humanas a lo largo de más de 6700 años (ca. 7400 - 650 años A.P.). Si bien existen materiales y fechados vinculados tanto a actividades de cazadores-recolectores muy tempranos como a eventos de grupos tardíos, el mayor corpus de evidencias se vincula a las ocupaciones agro-pastoriles del $1^{\circ}$ Milenio d. C. (intervalo 1850 1550 años $A P$ ), representadas por la asociación de sectores residenciales/domésticos (aldea) y áreas productivas agrícolas (andenes) y pastoriles (corrales) (Oliszewski et al. 2015). El presente trabajo tiene por objetivo exponer los estudios que se vienen llevando a cabo en relación a la tecnología cerámica del $1^{\circ}$ Milenio $d$. C. En ese sentido, se realiza una primera aproximación desde una perspectiva traceológica, donde se intenta observar a nivel local las técnicas de modelado a mano y sobre los posibles modos de hacer de los grupos que habitaron la quebrada para este período. Especificamente, la propuesta de trabajo se centra en el registro y estudio de macrotrazas de manufactura analizadas.
\end{abstract}

Palabras clave: El Infiernillo; Quebrada de Los Corrales; ${ }^{\circ}$ Milenio d.C.; Tecnología cerámica; Traceología

\begin{abstract}
Quebrada de Los Corrales is an archaeological area rich in evidence that account for diverse human occupations throughout more than 6700 years (ca. 7400 - 650 years B.P.). Although some dates and materials are linked to early hunting-gatherers activities as well as events pointing out to late groups, the largest body of evidence relates to the $1^{\text {st }}$ Millenium AD agro-pastoral occupations (1850 - 1550 years BP interval), represented by the association of residential/domestic (village) sector along with agriculture (terraces) and herding (corrals) areas (Oliszewski et al. 2015). This paper aims to present studies carried out on ceramic technology of the $1^{\text {st }}$ Millenium $a$. C. To this end a first approach is made from a traceology perspective, attempting to see hand-made shaping techniques and possible "savoir-fare" from groups of this period on a local scale. Specifically, the work proposal focuses on the study and recording of analyzed macro-traces.
\end{abstract}

Keywords: El Infiernillo; Quebrada de Los Corrales; 1st Milennium A.C.; Ceramic Technology; Traceology

Quebrada de Los Corrales (El Infiernillo, Tucumán), se encuentra ubicada por encima de los 3.000 msnm en el sector Norte del Sistema de las Sierras del Aconquija. Ocupa una superficie aproximada de $28 \mathrm{Km} 2$ y corresponde a la cuenca inferior, media y superior del río homónimo. Las investigaciones llevadas a cabo hasta el momento permitieron identificar evidencias arqueológicas correspondientes tanto al Holoceno medio como al Tardío (ca. 7.800 - 600 años AP) (Martínez et al. 2013, Oliszewski et al. 2015, 2018). Dentro de esta larga secuencia ocupacional de casi 6.700 años, fueron registrados numerosos sitios de distintas cronologías y funcionalidad, concentrando el mayor corpus de evidencias materiales el lapso temporal 1850 - 1550 años AP, relacionado con las ocupaciones agro-pastoriles del $1{ }^{\circ}$ Milenio D.C. Estas ocupaciones están conformadas por un gran núcleo aldeano de estructuras subcirculares compuestas y por aproximadamente 500 hectáreas de áreas productivas (andenes y corrales) (Oliszewski et al. 2015). (Figura 1). 
La aldea se encuentra agrupada en el área meridional de la quebrada hacia ambas márgenes del curso superior del río de Los Corrales, compuesta por al menos medio centenar de núcleos habitacionales "patrón Tafi" (sensu Berbarián y Nielsen 1988), llamado Puesto Viejo 1 y 2 (Di Lullo 2010, Oliszewski et al. 2015). Entre los materiales recuperados tanto en estratigrafía como en superficie los vestigios cerámicos se presentan abundantes y de diversas facturas, algunos diagnósticos por su decoración y asignables todos a estilos decorativos conocidos para el $1^{\circ}$ Milenio D.C. como Tafí, Candelaria, Ciénaga y Vaquerías (Gramajo Bühler 2009).

Teniendo en cuenta que en la Quebrada de Los Corrales (en adelante QDLC), existe una gran diversidad de evidencias arqueológicas asociadas a las ocupaciones agro-pastoriles y que hasta el momento no se han abordado estudios sistemáticos sobre la producción cerámica, la misma se presenta como una microrregión de gran potencial para estudiar distintos procesos desde múltiples perspectivas. En este sentido, este trabajo tiene por objetivo presentar algunos de los resultados obtenidos en relación a la tecnología cerámica del $1^{\circ}$ Milenio D.C., incorporando una primera aproximación desde una perspectiva traceológica sobre una muestra de fragmentos procedentes de Puesto Viejo 1 y 2 (en adelante PV1 y 2).

Desde esta perspectiva analítica, se intenta observar y discutir a nivel local sobre las diferentes maneras de hacer presentes en Puesto Viejo 1 y 2 en relación al modelado (Calvo Trías y García Rosselló 2011, 2014, García Rosselló 2018, Roux 2019, Petrequin y Petrequin 1999, Gosselain 2002), en nuestro caso, de los grupos que habitaron la quebrada (Gramajo Bühler 2009, 2013, 2018).

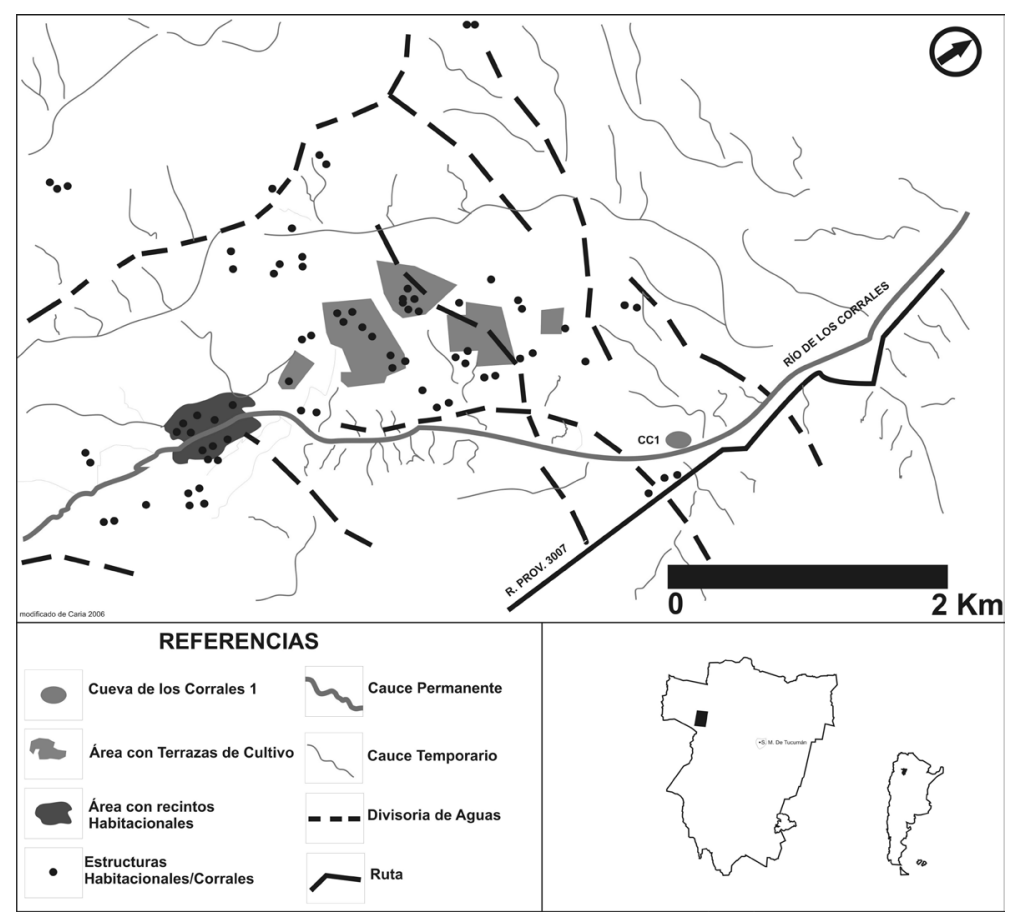

Específicamente, este trabajo se centra en el registro y estudio de macrotrazas de manufactura observadas y analizadas en fragmentos cerámicos de bordes, siguiendo la propuesta metodológica de Calvo Trías et al. (2004), García Rosselló (2010), García Rosselló y Calvo Trías (2013, 2019a, 2019b).

\section{Contextualización teórico-metodológica: análisis traceológico y savoir-fare}

Se propone aquí una aproximación a la tecnología cerámica entendida como el conjunto de conocimientos que los grupos humanos poseen para producir bienes socialmente valiosos (Lemonnier 1989), en donde las tecnologías no son vistas simplemente como medios usados por las sociedades para actuar sobre su ambiente físico (Lemonnier 1992), sino por el contrario, los sistemas tecnológicos deben ser entendidos como sistemas integrados a la estructura social en el que se desarrollan. Es decir, el estudio de los procesos tecnológicos supera el análisis del mero soporte físico para concentrarse íntimamente con los fenómenos sociales, ya que la práctica cotidiana de la fabricación, uso, intercambio y abandono de los objetos funciona inserta en un mundo históricamente contingente y complejo, y que por lo tanto la tecnología engloba las prácticas cotidianas sobre la realidad material, fruto del conjunto de relaciones sociales significadas y negociadas que estructuran, a su vez, dichas prácticas (Calvo Trías y García Rosselló 2014).

Desde esta perspectiva teórica, estudiar la manufactura de la cerámica nos posibilita reconocer distintos savoir-fare o modos de hacer que pueden englobar desde algunos gestos técnicos a secuencias de fabricación completas (Chevalier 1991; Karlin 1991; Petrequin y Petrequin 1999; Roux 2019). Los artesanos hacen elecciones técnicas, a modo de gestos, operaciones y técnicas, en las etapas de la secuencia de producción, y estas dan cuenta de conocimientos sobre la tradición y aprendizaje socialmente aceptados, que pasan de una generación a la próxima (Rye 1981; Stark 1999; Cremonte 2001; Gosselain 2002; Calvo Trías et al. 2015; Roux 2019). La repetición continua de gestos y secuencias técnicas en el tiempo y el espacio favorece la estabilización de formas específicas de savoir faire que reflejan la interacción existente entre los alfareros (Albero Santacreu 2014).

Figura 1. ubicación geográfica del área de estudio y de los sitios PV1 y 2 (aldea)

Figure 1. geographic location of study area and sites PV1 and 2 (village) 
El presente trabajo, se centra específicamente en el estudio del modelado de la cerámica. Entendiendo este proceso, según el diccionario de la Real Academia Española (2001) como "formar de cera, barro u otra materia blanda una figura u adorno". En términos generales, esta sería la acepción más aceptada por los investigadores y se podría concretar con la definición propuesta por Balfet et al. (1983) al entender que el modelado estaría compuesto por aquel conjunto de acciones que tienen por objetivo la consecución de la forma de la vasija a partir de la pasta arcillosa. Por su parte, Rye (1981: 58) utiliza una acepción más mecánicotécnica del término, al entender que el modelado sería la presión aplicada a la arcilla, lo que incluye variables relacionadas con la fuerza realizada, el área en la que se aplica dicha fuerza, así como las condiciones de la arcilla, especialmente aquellas relacionadas con el contenido del agua. Todo ello dará lugar a una deformación específica de la masa arcillosa. Otros autores distinguen entre el modelado propiamente dicho y los tratamientos de superficie (Orton et al. 1997), o entre el montaje y el acabado (Arnal 1986; Roux 1994, 2019). En la literatura anglosajona, para referirse a esta etapa, también se ha utilizado el término forming techniques (Rye 1981; Rice 1987; Orton et al. 1997) o Fashioning (Roux 2019) y en la francófona el de façonance (Livingstone 2007, Balfet et al. 1983 Arnal 1986).

El modelado, al contrario de otras etapas del proceso de fabricación, como la cocción y la obtención y preparación de la pasta, que son actividades y estrategias grupales, está más individualizada, al estar asociado a personas y tipos cerámicos concretos por lo que es mucho más fiable para identificar contactos sociales y estrategias comparativas (Gosselain 2002; Petrequin y Petrequin 1999). Del mismo modo, es una parte del proceso de fabricación mucho menos permeable a los cambios, debido, entre muchos otros factores, a su poca visibilidad social y afirmación identitaria, a diferencia de la forma o la decoración (García Rosselló 2011; Calvo Trías y García Rosselló 2011; García Rosselló 2018).

Desde que Helene Balfet publicará en 1953 Note sur le façonnage des poteries prehistoriques, el análisis traceológico para la identificación de los sistemas de modelado de las cerámicas se ha ido generalizando. Las reticencias iniciales se han ido superando en las últimas décadas gracias al esfuerzo de sistematización realizado a través de la confección de colecciones de referencia etnográficas y experimentales (ver un estado de la cuestión en García Rosselló y Calvo Trías 2019a), desarrollado por un reducido grupo de investigadores (Rye 1981; Balfet et al. 1983; Roux 1994, 2019 Huysecom 1992, 1994; Courty y Roux 1995; Pierret 1995; Martineau 2000; Gelbert 2003, 2005). Nuevas propuestas metodológicas e interpretativas han sido realizadas recientemente desde problemáticas estrictamente arqueológicas (Visseyrias 2007; García Rosselló 2010; Gomart 2014, Gomart et al. 2017; Colas
2016; Neumannová et al 2017; Cámara et al. 2017-2018).

Siguiendo las sistematizaciones precedentes, nuestra propuesta se fundamenta en el análisis traceológico de las marcas de manufactura (sensu García Rosselló 2010; García Rosselló y Calvo Trías 2013; 2019b). Estas (macrotrazas), son aquellas marcas de fabricación superficiales o visibles en la fractura transversal de la vasija, que tienen un origen tecnológico y que son observables a simple vista o con baja magnificación (García Rosselló y Calvos Trías 2013, 2019). Documentarlas, analizarlas y entenderlas en su contexto nos permite aproximarnos a los procesos de producción y, por lo tanto, reconstruir las técnicas de manufactura empleadas. A diferencia de otras aproximaciones metodológicas, nuestro análisis se orienta a la descripción morfológica de las marcas y el análisis del proceso de formación (directas o indirectas) como estrategia para el desarrollo del proceso inferencial de identificación de los gestos, operaciones y técnicas de manufactura.

El análisis de los sistemas de modelado y de cadena operativa a partir de las macrotrazas para el NOA, es un ámbito de investigación poco desarrollado hasta el momento, y solo se cuenta con los antecedentes de Wynveldt (2008), que analiza las huellas de manufactura macroscópicos de dos conjuntos de vasijas Belén del Valle de Hualfín (Catamarca), de Pérez Pieroni (2015), que aborda la manufactura cerámica Tardía y Colonial en la cuenca sur de Pozuelos y el área de Santa Catalina (Puna de Jujuy) y el de Vera y De La Fuente (2018), que realizan una primera aproximación a los estudios de traza sobre urnas funerarias estilo Saujil (Catamarca). En este sentido, este trabajo busca, además, complementar las investigaciones que se vienen realizando en la Quebrada de Los Corrales y para el periodo Formativo en torno a los estudios de los sistemas de modelado a mano de la cerámica, a partir de la identificación trazas.

\section{Materiales y Métodos}

La muestra analizada constó de un total de $\mathrm{N}=45$ fragmentos de bordes de recolección de superficie (prospección 2014), procedente de PV1 ( $\mathrm{N}=25)$ y PV2 $(\mathrm{N}=20)$ (Figura 2). Si bien la muestra original constó de un $\mathrm{N}=305$ fragmentos, para este trabajo se seleccionaron y analizaron solo aquellos bordes que pudieran servir para realizar una reconstrucción gráfica total o parcial de perfiles y con ello poder definir la morfología de la pieza (Balfet et al. 1983; García Rossselló 2010; Roux 2019). Asimismo, la cantidad de información que se obtiene depende de la orientación, longitud del arco y de la longitud total de cada uno de ellos. Por lo que, los bordes seleccionados debían representar, como mínimo, el 10\% o $30^{\circ}$ de abertura del recipiente (siguiendo la propuesta de Núñez Regueiro 2005) (Tabla 1).

Para llevar adelante los análisis aquí planteados, se 


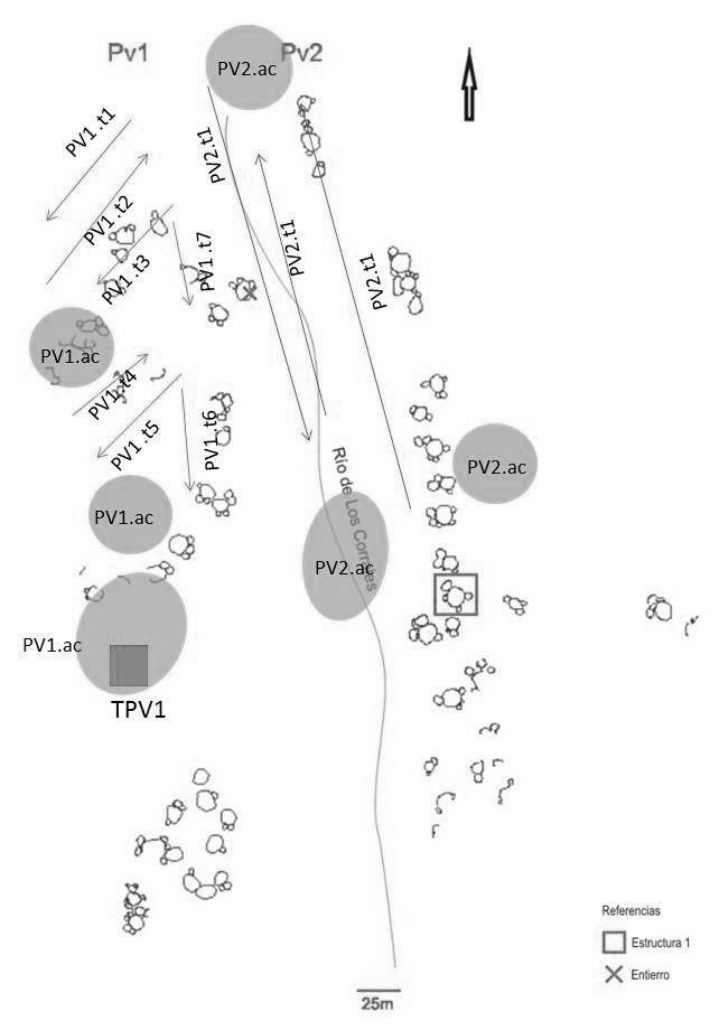

Figura 2. PV1 y 2: transectas realizadas en los distintos sectores. Las zonas demarcadas en grises señalan las áreas ciegas (ac), las cuales no pudieron ser prospectadas.

Figure 2. PV1 and 2: transects carried out in the different sectors. The areas demarcated in gray indicate the blind areas (ac), which could not be surveyed.

efectuaron observaciones a ojo desnudo y con lupa de mano de 30x de aumento.

La metodología adoptada para el análisis está basada en la propuesta de Calvo Trías et al. (2004), García Rosselló (2010) y García Rosselló y Calvo Trías (2013), la cual plantea que hay que definir las diferentes macrotrazas por separado e identificar las técnicas complejas a partir de la agrupación de trazas. Es decir, no a partir del sistema de fabricación adoptado, sino de la morfología de la macrotraza identificada, documentar sus atributos $y$, a partir de ellos, su origen y formación.

Siguiendo este planteamiento se realizó el análisis de los fragmentos de acuerdo a los siguientes niveles:

a) Familia: primer nivel de análisis, los cuales están definidos por sus características formales generales y por su proceso de formación. Pueden ser: 1) Traza de Formación Directa (F:TFD): se generan cuando el/la alfarero/a desarrolla la operación técnica en el momento que la arcilla es manipulable. 2) Traza de Formación Indirecta (F:TFI): se generan debido a fenómenos funcionales o postdepositacionales una vez que la pieza ya está cocida.
Tabla 1. Descripción de la muestra analizada. Según procedencia de los fragmentos, formas asociadas y grupo estilístico.

Table 1. Description of the analyzed sample. According to the procedence of the fragments, associated forms and stylistic group.

\begin{tabular}{|c|c|c|c|}
\hline \multicolumn{2}{|c|}{ Cantidad Frag. de Borde } & $\begin{array}{c}\text { Grup. Est. } \\
\text { Asociado }\end{array}$ & $\begin{array}{c}\text { Forma Asociada (sensu } \\
\text { Bugliani 2008 y Balfet } \\
\text { et al. 1993) }\end{array}$ \\
\hline PV1 & PV2 & Tafí & $\begin{array}{c}\text { Ollas Globulares a Sub } \\
\text { Globulares }\end{array}$ \\
\hline 4 & 7 & Candelaria & \\
\hline 10 & 3 & Candelaria & Cuencos \\
3 & 2 & Vaquerías & \\
\hline
\end{tabular}

b) Atributos: luego de definida la familia, se describe la forma y toda una serie de atributos que caracterizan las trazas (cualidades, característica o propiedades de la traza observada, distribución, sección, localización, disposición, estructura, etc.).

c) Procesos de Inferencia (PI): una vez definida la Familia, la morfología y los atributos asociados a cada traza se definen: 1) La Fase (F): clasificación de los diferentes estados físicos de la arcilla en relación a la pérdida de agua fruto de la manipulación realizada por el alfarero/a durante el proceso de fabricación de una vasija. Fase I a III: Modificación del estado físico de la arcilla (añadido de agua - manipulación). Fase l: estado natural, Fase II: mezcla y preparación y Fase III: estado fresco. Fase IV a X: no se puede modificar el estado físico de la arcilla (pérdida progresiva de agua - estabilización). Fase IV: primer secado, Fase V: textura cuero, Fase VI: segundo secado, Fase VII: estado seco, Fase VIII: cocción, Fase IX: enfriamiento y Fase $X$ : acabado. 2) El Proceso Tecnológico Marco (PTM): aquellos procesos necesarios e ineludibles que el alfarero/a debe realizar para fabricar una pieza cerámica. 3) El Proceso Tecnológico Marco Especifico (PTMEsp). 4) El Proceso Tecnológico Pormenorizado (PTP): cada una de las actuaciones realizadas durante la fabricación de una cerámica.

En base a lo observado durante el análisis de los fragmentos de bordes, se confeccionó una tabla, en donde se consignaron los distintos atributos y variables de las diferentes familias de traza para cada fragmento analizado. De esta forma se pudo sistematizar la información y realizar comparaciones entre ellos (Tabla 2).

\section{Resultados}

Del total de fragmentos ( $\mathrm{N}=45$ ), el $100 \%$ presentó dentro de F:TFI fracturas transversales por lo cual se pudo observar el Proceso Tecnológico Marco de modelado. Cabe aclarar que este tipo de fracturas dejan expuesto el perfil de la pieza, pudiendo en este sentido observar la forma en la cual fue levantada. A su vez, las fracturas 


\begin{tabular}{|c|c|c|c|c|c|c|c|c|c|}
\hline $\begin{array}{l}\text { Fragmento } \\
\mathrm{s}\end{array}$ & $F$ & Traza & Distrib. & PTM & PTMEsp. & PTP & Cara & Finalidad & $\begin{array}{l}\text { Fas } \\
\text { e }\end{array}$ \\
\hline \multirow{3}{*}{$\begin{array}{l}\text { PV1 (01 al } \\
05) \text { y PV2 } \\
\text { (01 al 05) }\end{array}$} & $\begin{array}{l}\text { F:TF } \\
D\end{array}$ & $\begin{array}{l}\text { Bandas } \\
\text { Homogeneas } \\
\text { paraleles }\end{array}$ & Continua & $\begin{array}{l}\text { trat. de } \\
\text { Sup. }\end{array}$ & fricción & $\begin{array}{l}\text { Homogenizació } \\
\text { n de la Sup. }\end{array}$ & \multirow{3}{*}{$\begin{array}{l}\text { Ext - } \\
\text { Inter }\end{array}$} & $\begin{array}{l}\text { Trat. Final } \\
\text { de Sup. - } \\
\text { acabado } \\
\text { de Sup. }\end{array}$ & \multirow{3}{*}{$\begin{array}{l}\text { III } \\
y \\
\text { V }\end{array}$} \\
\hline & $\mathrm{F}: \mathrm{TFI}$ & $\begin{array}{l}\text { Frac. } \\
\text { transversal }\end{array}$ & & modelado & $\begin{array}{l}\text { tec. de } \\
\text { formación }\end{array}$ & $\begin{array}{l}\text { urdido cabalg. } \\
\text { Ext. }\end{array}$ & & $\begin{array}{l}\text { Confecció } \\
n\end{array}$ & \\
\hline & F:TFI & Frac. Hrz. & & modelado & $\begin{array}{l}\text { tec. de } \\
\text { formación }\end{array}$ & urdido & & $\begin{array}{c}\text { Confecció } \\
\mathrm{n}\end{array}$ & \\
\hline \multirow{3}{*}{$\begin{array}{l}\text { PV1 (06 al } \\
12) \text { y PV2 } \\
\text { (06 al 12) }\end{array}$} & $\begin{array}{l}\text { F:TF } \\
D\end{array}$ & $\begin{array}{l}\text { Bandas } \\
\text { Homogeneas }\end{array}$ & Continua & $\begin{array}{l}\text { trat de } \\
\text { Sup. }\end{array}$ & fricción & $\begin{array}{l}\text { Homogenizació } \\
\text { n de la Sup. }\end{array}$ & \multirow{3}{*}{$\begin{array}{l}\text { Ext - } \\
\text { Inter }\end{array}$} & $\begin{array}{l}\text { Trat. Final } \\
\text { de Sup. - } \\
\text { acabado } \\
\text { de Sup. }\end{array}$ & \multirow{3}{*}{$\begin{array}{l}\text { III } \\
\text { y } \\
\text { V }\end{array}$} \\
\hline & $\mathrm{F}: \mathrm{TFI}$ & $\begin{array}{l}\text { Frac. } \\
\text { transversal }\end{array}$ & & modelado & $\begin{array}{l}\text { tec. de } \\
\text { formación }\end{array}$ & $\begin{array}{l}\text { urdido cabalg. } \\
\text { Ext. }\end{array}$ & & $\begin{array}{l}\text { Confecció } \\
\text { n }\end{array}$ & \\
\hline & F:TFI & Frac. Hrz. & & modelado & $\begin{array}{l}\text { tec. de } \\
\text { formación }\end{array}$ & urdido & & $\begin{array}{c}\text { Confecció } \\
n\end{array}$ & \\
\hline \multirow{2}{*}{$\begin{array}{l}\text { PV1 (13 al } \\
18) \text { y PV2 } \\
(13 \text { al 15) }\end{array}$} & $\begin{array}{l}\text { F:TF } \\
D\end{array}$ & $\begin{array}{l}\text { Bandas } \\
\text { Homogeneas }\end{array}$ & Continua & $\begin{array}{l}\text { trat de } \\
\text { Sup. }\end{array}$ & fricción & $\begin{array}{l}\text { Homogenizació } \\
\mathrm{n} \text { de la Sup. }\end{array}$ & \multirow{2}{*}{$\begin{array}{l}\text { Ext - } \\
\text { Inter }\end{array}$} & $\begin{array}{l}\text { Trat. Final } \\
\text { de Sup. - } \\
\text { acabado } \\
\text { de Sup. } \\
\end{array}$ & \multirow{2}{*}{$\begin{array}{l}\text { III } \\
\text { y } \\
\text { V }\end{array}$} \\
\hline & F:TFI & $\begin{array}{l}\text { Frac. } \\
\text { transversal }\end{array}$ & & modelado & $\begin{array}{l}\text { tec. de } \\
\text { formación }\end{array}$ & $\begin{array}{l}\text { urdido cabalg. } \\
\text { Ext. }\end{array}$ & & $\begin{array}{l}\text { Confecció } \\
n\end{array}$ & \\
\hline \multirow{3}{*}{$\begin{array}{l}\text { PV1 (19 al } \\
21) \text { y PV2 } \\
(16 \text { y 17) }\end{array}$} & TFD & $\begin{array}{l}\text { alisado de } \\
\text { superficie }\end{array}$ & continua & $\begin{array}{l}\text { Tratamient } \\
\text { o Sup. }\end{array}$ & compresión & $\begin{array}{l}\text { Homogenizació } \\
\mathrm{n} \text { de la Sup. }\end{array}$ & $\begin{array}{l}\text { Ext.- } \\
\text { Inte. }\end{array}$ & $\begin{array}{l}\text { trat. final } \\
\text { de supe. } \\
\text { Acabado } \\
\text { de sup. }\end{array}$ & \multirow{3}{*}{$\begin{array}{l}\text { III } \\
\text { y } \\
\text { V }\end{array}$} \\
\hline & TFI & $\begin{array}{l}\text { superposición } \\
\text { y exfoliación }\end{array}$ & $\begin{array}{l}\text { discontinua } \\
\mathrm{s}\end{array}$ & $\begin{array}{l}\text { Tratamient } \\
\text { o Sup. }\end{array}$ & adición & pintado & Inte. & $\begin{array}{l}\text { trat. final } \\
\text { de sup. } \\
\text { Acabado } \\
\text { de sup. }\end{array}$ & \\
\hline & TFI & $\begin{array}{l}\text { fractura } \\
\text { transversal }\end{array}$ & & Modelado & $\begin{array}{l}\text { Técnica de } \\
\text { formación }\end{array}$ & indeterm. & & $\begin{array}{l}\text { Confecció } \\
n\end{array}$ & \\
\hline \multirow{5}{*}{$\begin{array}{l}\text { PV1 (22 al } \\
25) \text { y PV2 } \\
(18 \text { y } 20)\end{array}$} & TFD & $\begin{array}{l}\text { Bandas } \\
\text { heterogeneas }\end{array}$ & $\begin{array}{l}\text { discontinua } \\
\mathrm{s}\end{array}$ & $\begin{array}{l}\text { Tratamient } \\
\text { o Sup. }\end{array}$ & fricción & $\begin{array}{l}\text { Homogenizació } \\
\mathrm{n} \text { de la Sup. }\end{array}$ & Ext. & $\begin{array}{l}\text { trat. final } \\
\text { de sup. } \\
\text { Acabado } \\
\text { de sup. }\end{array}$ & \multirow{5}{*}{$\begin{array}{l}\text { II } \\
y \\
\text { VI }\end{array}$} \\
\hline & TFD & $\begin{array}{l}\text { Bandas } \\
\text { heterogeneas }\end{array}$ & continua & Trat. Sup. & fricción & $\begin{array}{l}\text { Homogenizació } \\
\mathrm{n} \text { de la Sup. }\end{array}$ & Inte. & $\begin{array}{l}\text { trat. final } \\
\text { de sup. } \\
\text { Acabado } \\
\text { de sup. }\end{array}$ & \\
\hline & TFI & $\begin{array}{l}\text { fractura } \\
\text { transversal }\end{array}$ & & Modelado & $\begin{array}{l}\text { Técnica de } \\
\text { formación }\end{array}$ & $\begin{array}{l}\text { urdido cabalg. } \\
\text { Ext. }\end{array}$ & $\begin{array}{l}\text { Ext. - } \\
\text { Inte }\end{array}$ & $\begin{array}{l}\text { Confecció } \\
\mathrm{n}\end{array}$ & \\
\hline & TFI & $\begin{array}{l}\text { superposición } \\
\text { y exfoliación }\end{array}$ & $\begin{array}{l}\text { discontinua } \\
\mathrm{s}\end{array}$ & Trat. Sup. & adición & engobe rojo & Inte. & $\begin{array}{l}\text { trat. final } \\
\text { sup. } \\
\text { Acabado } \\
\text { de sup. } \\
\end{array}$ & \\
\hline & TFI & \begin{tabular}{|l|} 
Fractura \\
Lineal
\end{tabular} & & Modelado & $\begin{array}{l}\text { Téc. de } \\
\text { formación }\end{array}$ & urdido & $\begin{array}{l}\text { Ext. - } \\
\text { Inte }\end{array}$ & $\begin{array}{l}\text { Confecció } \\
\mathrm{n}\end{array}$ & \\
\hline
\end{tabular}

Tabla 2. Procedencia de la muestra y descripción de las trazas analizadas para cada fragmento. Se agruparon aquellos fragmentos que compartían ciertas Trazas de Formación Directa e Indirecta como el Proceso Tecnológico Marco Específico, su distribución, ubicación y su Proceso Tecnológico Pormenorizado (tipo de modelado).

Table 2. Origin of the sample and description of the traces analyzed for each fragment. Those fragments that shared certain Traces of Direct and Indirect Training were grouped, such as the Specific Framework Technological Process, its distribution, location and its Detailed Technological Process (type of modeling).

aquellos fragmentos en donde se pudo determinar el proceso de modelado de aquellos que no. Es decir, todos los fragmentos que presentaron un acabado de superficie alisado y pulido (PTP), con un PTMEsp. de fricción, están relacionados a F:TFI urdido por cabalgadura externa, y aquellos fragmentos que presentaron un alisado-pintado (PTP), están relacionados a $F: T F I$ indeterminados. En este sentido podemos decir que el $88,8 \%$ de la muestra analizada

o grietas con un patrón longitudinal u horizontal (correspondiente a la F:TFI), se encontraron presentes en el $75 \%$ de los fragmentos analizados. Estas pueden ser interpretadas como evidencia del modelado mediante superposición de rollos de arcilla (García Rosselló 2010; García Rosselló 2019b) (Figura 3).

Al comparar ambas trazas de formación indirecta, podemos llegar a inferir el Proceso Tecnológico Pormenorizado, en el cual se ha podido observar que el $88.8 \%$ presenta un modelado por urdido en cabalgadura externa y el restante $11.2 \%$ indeterminado. Del total de fragmentos asociados a un modelado por cabalgadura externa, el 55\% proviene de PV1 y el restante 45\% a PV2. Por su lado, del total de los fragmentos indeterminados, el $60 \%$ proviene del PV1 y el restante $40 \%$ de PV2.

Cuando analizamos F:TFD (finalidad de acabado de superficie), vemos que se presenta una uniformidad en presenta un sistema de urdido por cabalgadura externa (los rodetes se colocan desde la pared exterior de la vasija y se aplican solo parcialmente sobre el colombino arrastrando el resto de la arcilla sobre la pared (Livingstone 2007; García Rosselló y Calvo 2013) y un acabado de superficie alisado o pulido, y el restante 11,2\% son fragmentos pintados en donde no se ha podido determinar el tipo de urdido.

Dentro de las diferencias observables al tipo de trazas registradas, vemos que el 41,66\% corresponden a bandas homogéneas paralelas ( $F: T F D$ finalidad tratamiento final de la superficie), el 33,33\% alisado de superficie y el 25\% a bandas heterogéneas (paralelas y diagonales). En cuanto a la distribución de las mismas, el $75 \%$ se presentan continuas y el $25 \%$ discontinuas. En términos generales, se observó una tendencia de aquellos fragmentos que presentaban bandas homogéneas paralelas a poseer una ubicación exterior e interior (58,33\%), los que 


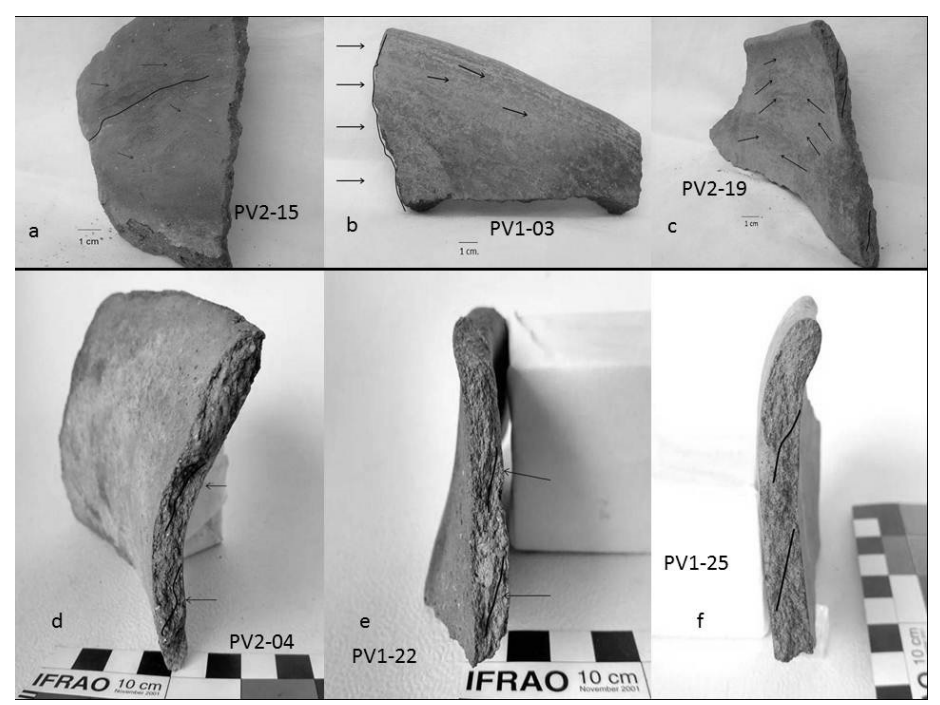

Figura 3. Representación de algunas de las trazas registradas. a) fractura transversal (TFI). Se muestra el sentido de las bandas heterogéneas paralelas (TFD) y PTMEsp por compresión. b) fractura trasversal (TFI), bandas homogéneas paralelas (TFD). c) fractura transversal (TFI) urdido cabalgadura externa .bandas paralelas-diagonal (TFD). d,e y f) se muestra las fracturas transversales y se marca la forma de levantado, urdido por cabalgadura externa (TFI).

Figure 3. Representation of some of the traces recorded. a) transverse fracture (TFI). The direction of the parallel heterogeneous bands (TFD) and PTMEsp by compression is shown. b) transverse fracture (TFI), parallel homogeneous bands (TFD). c) transverse fracture (TFI) warped by external cape. parallel-diagonal bands (TFD). $d$, e and $f$ ) the transverse fractures are shown and the raised shape is marked, concocted by external fit (TFI). presentaban un alisado de superficie, cara exterior $(16,66 \%)$ y los que presentaban bandas heterogéneas, exterior e interior (25\%).

Es interesante destacar que este tipo de trazas tecnológicas son significativas (sensu Calvo Trías et al. 2004), ya que son huellas presentes en la superficie de la pasta que nos pueden informar sobre los procesos de fabricación de la pieza. En este sentido, la identificación y análisis de las bandas nos permite por un lado contrastar el estado de la pasta al momento en que se realizó dicha traza, siendo características su realización en el estado de textura de cuero (Fase $V$, estadio donde puede manipularse únicamente la superficie de la arcilla al estar todavía húmeda, a la vez que la presión sobre la pasta la puede deformar y fracturar irreversiblemente), y por otro, tratar de identificar si existe o no alguna estandarización en cuanto a las mismas.

Siguiendo la propuesta de Gramajo Bühler (2009, 2018), en la cual plantea que los grupos estilísticos registrados para QDLC estarían conformados por Tafí, Candelaria y Vaquerías, vemos que aquellos fragmentos que presentaron un urdido por superposición de rollos de arcilla con cabalgadura externa están asociados a los grupos estilísticos Tafí y Candelaria. Por su parte, los fragmentos pintados, en los cuales no se pudo identificar el tipo de superposición de rollos de arcillas están asociados al grupo estilístico Vaquerías (Figura 4).

Las formas registradas (de manera tentativa a partir de los bordes analizados, y siguiendo lo planteado por Bugliani 2008) varían entre:

a) ollas globulares a subglobulares medianas a grandes de borde recto a borde evertido de cocción oxidante. Recipientes cerrados, restringidos o pocos restringidos respecto del diámetro de la abertura, con cuellos cortos o sin ellos y donde el diámetro mínimo es superior o igual a un tercio del diámetro máximo (Balfet et al. 1983). El $100 \%(\mathrm{~N}=22)$ de los fragmentos asignados a estas formas presentan modelado por cabalgadura externa.

b) Cuencos: medianos a pequeños, de formas abiertas, cocción reductora/oxidante de pasta fina. Son recipientes donde el diámetro de la abertura (boca) está comprendido entre una vez y media y dos veces y media de la altura (Balfet et al. 1983).

Del total de los fragmentos asignados a estas formas $(\mathrm{N}=$ 23), el $78,23 \%$ presentan modelado por cabalgadura externa y el restante $21,77 \%$ a indeterminados.

Cuando comparamos las formas presentes con el tipo de levantado, vemos que el modelado por cabalgadura externa está presente en todas las formas que se han registrado hasta el momento en QDLC (Gramajo Bühler 2009, 2018), siendo solo en el caso de algunos cuencos, caracterizados por estar asociados al grupo estilístico Vaquerías, en donde posiblemente se haya usado otra forma de modelado a mano que no sea el antes mencionado. Si bien los procesos de acabado de superficie pueden obliterar o invisibilizar las marcas de modelado de los pasos previos en la secuencia de producción (Rye 1981), cuando se analizan las fracturas transversales en los fragmentos (TFI), se puede observar este proceso de modelado.

Para el caso de los fragmentos asignados al grupo estilístico Vaquerías, no se ha podido identificar el proceso de modelado, pero sin embargo se ha podido observar una regularidad, que los diferencia del resto de fragmentos analizados, en cuanto a la confección de una traza tecnológica significativa como son las bandas. A diferencias del resto, son los únicos fragmentos que presentan bandas heterogéneas, tanto en su cara interna (continua) como externa (discontinua) (Tabla 2). Coincidimos con Lemonnier (1992), en que ninguna técnica debe ser concebida como un mero gesto, sino como una representación física de elecciones y esquemas mentales aprendidos a través de la tradición tecnología 


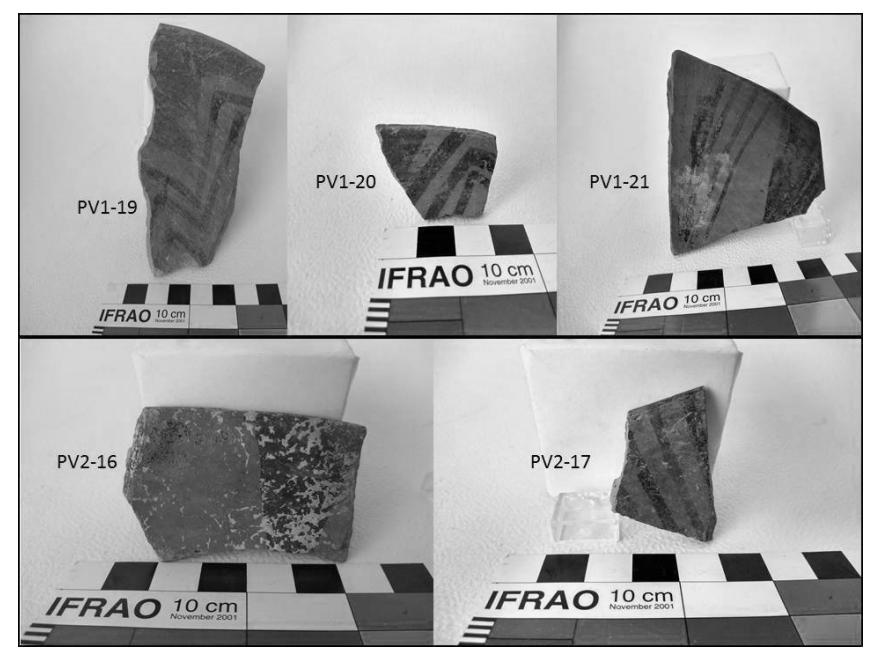

donde están inmersos y relacionados con la manera de trabajar del grupo y su contexto social, por lo que esta diferencia en cuanto a una traza nos podría estar hablando también posiblemente de modos de hacer distinto al resto del material analizado.

\section{Consideraciones Finales}

Identificar los sistemas de fabricación es únicamente un primer paso en el proceso interpretativo ya que el análisis tecnológico nos permite, además de aproximarnos al conocimiento de cómo se fabrican las cosas, comprender o inferir los aspectos sociales e ideológicos de los grupos que las produjeron. Es por lo tanto que este trabajo no pretende llegar a conclusiones acabadas, sino por lo contrario, empezar a generar preguntas sobre las posibles tradiciones tecnológicas presentes para QDLC.

En este sentido, y teniendo en cuenta los resultados obtenidos sobre el conjunto analizado, se plantea una marcada tendencia $(88,8 \%)$ en la operación técnica vinculada al levantado de la pieza, donde se evidencia el proceso tecnológico por urdimbre en cabalgadura externa en todas las formas registradas. El restante $11.2 \%$ de los fragmentos analizados están asociados solo a formas descriptas como cuencos. Estos han sido relacionados por Gramajo Bühler $(2009$, 2018) como una cerámica de cocción oxidante, de pasta fina, pintada, de formas abiertas, pequeñas, asociada al grupo estilístico vaquerías.

Es interesante destacar lo planteado por Korstanje (1998) y Pantorrilla Rivas (2009) en donde vinculan a la cerámica Vaquerías con una amplia circulación en el NOA e incluso al Norte de Chile y Sur de Bolivia. A su vez desde los análisis petrográficos y de procedencia de materia prima (Cremonte 1996, Bugliani y Pereyra Domingorena 2012, Cremonte y Pereyra Domingorena 2013, Guista 2015) plantean que no es de producción local para el área. En este sentido, los/as autores/as proponen como hipótesis que esta cerámica no fue manufacturada en la zona
Figura 4. Fragmentos de bordes Vaquerías. a) procedente de PV1. b) procedentes de PV2. En todos los casos no se pudo determinar el tipo de urdido.

Figure 4. Vaquerías edge fragments. a) from PV1. b) from PV2. In all cases the type of warp could not be determined.

donde fue hallada.

Para QDLC la abundancia de fragmentos Vaquerías es destacable en relación a otros sitios aledaños (Valle de Hualfín: Sitio 1 y 2 del Río Diablo, V. del Bolsón: La Mesada, V. del Cajón: Cardonal; Bordo Marcial, V. de Santa María: San José; Soria 2, V. de Tafí: El Mollar; Río las Piedras, Amaicha: Bajo Los Cardones, Quebrada del Toro: Las Cuevas, Tres Cruces, entre otros), sin embargo, cuando los comparamos con el resto de la muestra analizada, vemos que es la única que no podría presentar un proceso tecnológico por urdimbre en cabalgadura externa y que a su vez, presenta una posible estandarización en cuanto a la confección de una traza tecnológica significativa como ser las bandas, por lo tanto posiblemente de un modo de hacer distinto al resto de la muestra. Este dato, no es menor a la hora de pensarlas también como piezas que posiblemente sean de fabricación no local, y que por lo tanto respondan a tradiciones tecnológicas distintas a las existentes para la quebrada.

Consideramos, como hemos comentado, que el modelado es la parte de la secuencia de fabricación más resistente a los cambios y el cual requiere un proceso de aprendizaje más largo (ver también Gosselain 2002, García Rosselló 2011, 2018, 2019a). Esto obedece a que, en contextos de producción doméstica, las secuencias operacionales se trasmiten de generación en generación a través de un aprendizaje dentro del grupo familiar. Si además entendemos que no pueden existir hombres/mujeres sin objetos y objetos sin seres humanos, entendemos que la cultura material no puede ser concebida como un objeto neutro/pasivo, sino por el contrario activa (Dobres 2000; Dobres y Robb 2005). En este sentido, los objetos no solo son un reflejo de la sociedad que los produjo, sino también, un agente y/o constructor de la sociedad (Hodder 2012).

Por lo antes dicho, detectar, por ejemplo, la forma de cabalgamiento de los rollos en el levantado/modelado de la pieza cerámica nos puede dar una pauta para tratar de inferir la existencia del savoir-fare. Planteamos de manera tentativa que los habitantes de QDLC para el $1^{\circ}$ Milenio D.C. (ca.1850 - 1550 años AP) compartieron un mismo savoir-fare en el modelado por urdimbre de cabalgamiento externo para vasijas globulares a subglobulares de pasta intermedia gruesa a intermedia fina de cocción oxidante y reductora, como así también, las asociadas a formas abiertas, pequeñas, de base cóncava convexa. Pero, también coexistieron con otras producciones cerámicas 
no locales, enfocada particularmente a la cerámica Vaquerías.

No cabe duda que este tipo de inferencias no pueden estar descontextualizadas de los procesos que se estuvieron dando a nivel regional, ni tener en cuenta otros tipos de formas y pastas cerámicas características del periodo Formativo para el NOA. Sin embargo, este tipo de análisis nos demuestran la potencialidad que poseen a la hora de caracterizar un determinado grupo, no solo por su materialidad, sino también por las estrategias cognitivas tecnológicas que realiza y que produce la cultura material y que puede ser visualizada a través de la documentación de la cadena operativa. De hecho, algunos autores como Petrequin y Petrequin (1999) o Gosselain (2002) han demostrado que el papel social en el proceso de montaje y la transmisión del conocimiento técnico resulta de suma importancia, más que las formas o estilos de las cerámicas, para identificar grupos y contactos entre comunidades.

Los Nogales (Tucumán), 24 de Diciembre 2019

\section{Bibliografía}

Albero Santacreu, D. (2014) Materiality, Techniques and Society in Pottery Production. The Technological Study of Archaeological Ceramics through Paste Analysis. Varsovia, Polonia: De Gruyter.

Arnal, G. (1986). La céramologie préhistorique expérimentale. Archaeologie expérimentale, Archaedrome 2, cahier 2: 5-36.

Balfet, H. (1953). Note sur le façonnage des poteries prehistoriques. Bulletin de la Societe Prehistorique francaise $\mathrm{L}$, pp. 211-217.

Balfet, H. ; Fauvet, M. F. ; Monzon, S. (1983). Lexique et typologie des poteries. Paris, Francia: Presses du CNRS.

Berberián, E. y Nielsen, A. (1988). Análisis funcional de una unidad doméstica de la etapa formativa en el Valle de Tafí. E. Berberián (ed), Sistemas de asentamiento prehispánicos en la etapa formativa del Valle de Tafí, pp. 53-67. Córdoba, Argentina: Comechingonia,

Bugliani, M.F. (2008). Consumo y representación en el sur de los valles Calchaquíes (Noroeste argentino): Los conjuntos cerámicos de las aldeas del primer milenio A.D. Oxford, UK: BAR (British Archaeological Reports, International Series, S1774)

Bugliani, M. F y Pereyra Domingorena, L. (2012). Una aproximación estilístico-tecnológica a la cerámica polícroma "Vaquerías" del Noroeste Argentino. Estudios Atacameños 43:121-138.

Calvo Trías, M., Fornés Bisquerra, J., García Rosselló, J.,
Guerrero Ayuso, V., Juncosa Vicchierini, E., Quintana Abraham, C. y Salva Simonet, B. (2004). La Cerámica prehistórica a Mano: una propuesta para su estudio. Palma de Mallorca, España: El Tall.

Calvo Trías, M. y García Rosselló, J. (2011). Tradición técnica y contactos: un marco de reflexión centrado en la producción cerámica. Rubricatum 5: 393 - 401.

Calvo Trías, M. y García Rosselló, J. (2014). Acción Técnica, Interacción Social y Práctica Cotidiana: propuesta interpretativa de la tecnología. En Trabajos de Prehistoria 71 (1): 722.

Calvo Trías, M.; García Rosselló, J.; Javaloyas Molina, D.; Albero Santacreu, D. (2015): Playing with mud? An ethnoarchaeological approach to children's learning in Kusasi ceramic production. En M. Sánchez Romero; E. Alarcón García; G. Aranda Jiménez (eds.) Children, identity and space., SSCIP Monograph Series 4:88-104.,Oxford, UK: Oxbow.

Cámara, J., Clop, X., García, J., Pons, E., Saña, M. (20172018). Els processos de fabricació de ceràmiques a la Dou (Vall d'en Bas, La Garrotxa) durant el Bronze final (1290-920 cal ANE). Primers resultats. Cypsela 21: 43-66.

Chevallier, D. (1991) (ed.). Savoir-faire et Pouvoir transmettre. Transmission et apprentissage des savoirfaire et des techniques. Paris, Francia: Editions de la Maisons des Sciences de I'Homme.

Colas, C. (2016). Regard technique sur les poteries du Chasséen entre 4500 et 3500 ans Avant J. C. en Auvergne et dans le Bassin parisien à Travers le prisme de la coupe carénée. En Perrin, T., Chambon, P., Gijaba, J., Goude, G. (Eds.). Le Chasséen, des Chasséens. Retour sur une culture nationale et ses parallèles, pp. 18-20. Paris, Francia: Sepulcres de fossa, Cortaillod.

Courty, M. A. y Roux, V. (1995) Identification of Wheel Throwing on the basis of Ceramic Surface Features and Microfabrics, Journal of Archaeological Science 22: 1750.

Cremonte, M. B. (1996). De las pastas a los olleros del pasado. Volumen XXV Aniversario Museo Arqueológico Eduardo Casanova, pp. 47-53. Tilcara, Argentina: Instituto Interdisciplinario Tilcara, FFyL, UBA.

Cremonte, M. B. (2001). Las pastas cerámicas como una contribución a los estudios de identidad. Actas del XIII Congreso Nacional de Arqueología Argentina, (2): 199210. Córdoba.

Cremonte M. B y Pereyra Domingorena, L. (2013). Atlas de Pastas Cerámicas Arqueológicas. Petrografía de Estilos Alfareros del NOA. Universidad Nacional de Jujuy. EdiUnju. 
Di Lullo, E. (2010). El Espacio Residencial Durante el 1er Milenio d.C. en la Quebrada de Los Corrales (El Infiernillo, Tucumán). Tesis de grado no publicada, Facultad de Ciencias Naturales e IML, Universidad Nacional de Tucumán, Tucumán.

Dobres, M. A. (2000). Technology and social agency. Londres, UK: Blackwell.

Dobres, M. y Robb, J (2005) Doing Agency: Introductory Remarks on Methodology, Journal of Archaeological Method and Theory 12 (3): 159-166.

García Rosselló, J. (2010). Análisis traceológico de la cerámica: modelado y espacio social durante el Postalayotico (V-I a.C.) en la península de Santa Ponça (Calviá, Mallorca). Tesis doctoral. Universidad de las Islas Baleares. Mallorca, España.

García Rosselló, J. (2011). Modelado, aprendizaje y espacio social: una reflexión desde la tecnología cerámica. Revista Werkén 14: 63-74.

García Rosselló, J. (2018). Práctica Técnica y Práctica Social en la Producción Cerámica del Centro de Chile. Menga. Revista de Prehistoria de Andalucía. Monográfico 4:5974.

García Rosselló J y Calvo Trias M. (2013). Algo más que una vasija: modelado y espacio social en la producción cerámica. Actas del l Congreso Internacional Sobre Estudios Cerámicos, 359 - 392. Cadiz, España.

García Rosselló, J. y Calvo Trías, M. (2019a). Etnoarqueología como experimentación: propuesta para la interpretación de los patrones de fractura. Treballs d'Arqueologia, 23:181-202.

García Rosselló, J. y Calvo Trías, M. (2019b). Un análisis crítico de las colecciones de referencia etnográficas para el estudio del modelado. Treballs d'Arqueologia, 23:223-236.

Gelbert, A. (2003) Traditions cerámiques et emprentes techniques. Etude Etnoarcheologique dans le haute et moyenne vallées du fleuve Sénegal. París, Francia : Editions de la MSH, Epistèmes.

Gelbert, A. (2005) Reconnaissance des techniques et des méthodes de façonnage par l'analyse des macrotraces: êtude Ethnoarchéologique dans la valleé du Sénegal. En A. Livingstone Smith, D. Bosquet, R. Martineau (eds.), Pottery manufacturing processes: reconstitution and interpretation, (1349: 67-78).Oxford, UK : BAR International Series.

Giusta, M, N. (2015). La Alfarería en la Quebrada de Amaicha (Dpto. de Tafí del Valle, Provincia de Tucumán) durante el Primer Milenio A. D.: aportes sobre aspectos tecnológicos y fuentes de materia prima (Tesinia de Grado). Facultad de Ciencias Naturales e IML, UN, Tucumán, Argentina.

Gomart, L. (2014). Traditions Techniques \& Production. Céramique au Néolithique Ancien. Étude de huit sites rubanés du nord-est de la France et de Belgique. Leiden, Hollande: Sidestone Press.

Gomart, L.; Weiner, A.; Gabriele, M.; Durrenmath, G.; Sorin, S.; Angeli, L., Colombo, M.; Fabbri, C.; Maggi, R.; Panelli, C.; Pisani, D.; Radi, G.; Tozzi, C.; Binder, D. (2017). Spiralled patchwork in pottery manufacture and the introduction of farming to Southern Europe. Antiquity 91: 1501-1514.

Gosselain, O. P. (2002): Poteries du Cameroun Meridional. Styles, Techniques et raports a l'identité. París, France:CNRS.

Gramajo Bühler, C. M. (2009). Primera caracterización del conjunto cerámico de Quebrada de Los Corrales (El Infiernillo, Tucumán). Serie Monográfica y Didáctica 48: 121.

Gramajo Bühler, C. M. (2013). La cerámica fragmentaría como indicador de estabilidad del registro arqueológico: el sitio TPV1 (El Infiernillo, Tucumán) como caso de estudio. Serie Monográfica y Didáctica 53: 33.

Gramajo Bühler, C. M. (2018). Una Caracterización a la Cerámica de la Quebrada de Los Corrales (El Infiernillo, Tucumán). Aproximaciones Necesarias. Actas de la Segundas Jornadas Ñaupa AMAICHA, pp. 57-58. Amaicha del Valle, Tucumán, Argentina.

Hodder I. (2012). Entangled: An Archaeology of the Relationships Between Humans and Things. Chichester, UK Wiley- Blackwell.

Huysecom, E. (1992). Les percutors d'argile: des outils de potières africanes utilisés de la préhistorie a nos jours. Bulletin du Centre genevois d'anthropologie 3, 71-98.

Huysecom, E. (1994). Identification technique des céramiques africaines .En D. Binde and J. Courtin (eds.) Terre cuite et société: la céramique, document technique, économique (pp 31-44). París, France :Juan-les-Pins: Éditions APDCA.

Korstanje M. A. (1998). Desempolvando antigüedades: Consideraciones sobre el repertorio cerámico Vaquerías. Mundo de Antes 1, 69-117.

Lemonnier, P. (1989). Towards an anthropology of technology. Man 24, 526-527. 
Lemonnier, P. (1992). Elements for an anthropology of technology. Michigan, USA: Anthropological pepers 88, University of Michigan, Museum of Anthropology.

Livingstone, A. (2007). Chaîne Opératoire de la Poterie, Références ethnographiques, analyses et reconstitution. Tervuren, Belgique: Musée Royal de l'Afrique Centrale,

Karlin, C., (1991) Connaisance et savoir-faire: comment analiser un processus technique en Prehistoire. En R. Mora, X. Terradas, A. Parpal, C. Plana (eds.), Tecnología y cadenas operativas Líticas (pp:5-18). Bordeaux, France: Reunión Internacional, Treballs d'Arqueología.

Martineau, R. (2000). Poterie, techniques et sociétés. Études analytiques et expérimentales à Chalain et Clairvaux (Jura), entre 3200 et 2900 av. J.-C-. (Thèse de doctorat). Université de Franche-Comté. Dijon, France.

Martínez, J.G., Mauri, E., Mercuri, C., Caria, M. y Oliszewski, N. (2013). Mid-Holocene human occupations in Tucumán (Northwest of Argentina). Quaternary International. Human populations and environments during the mid-Holocene in the South-Central Andes.307: 86-95

Neumannová, K.; Petřík, J.; Vostrovská, I.; Dvořak, J.; Zikmund, T.; Kaiser, J. (2017). Variability in coiling technique in LBK pottery inferred by experiments and pore structure micro-tomography analysis. Archeologické rozhledy, 69:172-186.

Núñez Regueiro, V. (2005). Arqueometría para la reconstrucción de vasijas. Actas del I Congreso de Arqueometría, pp. 109. Rosario.

Oliszewski, N., Martínez, J. G., Di Lullo, E., Gramajo Bühler, C. M., Arreguez, G., Cruz, H., Mauri, E., Mercuri, C., Muntaner, A. y Srur, G. (2015). Contribuciones al Estudio de Sociedades Aldeanas en el Noroeste Argentino: El Caso de La Quebrada de Los Corrales (El Infiernillo, Tucumán). Korstanje, A.; M., Lazzari; M., Basile; F., Bugliani; V., Lema; L., Pereyra Domingorena y M., Quesada (eds.), Crónicas Materiales Precolombinas. Arqueología de los Primeros Poblados del Noroeste Argentino (pp 51-79). Buenos Aires, Argentina: Sociedad Argentina de Antropología.

Oliszewski, N., Martínez, J.G., Arreguez, G., Gramajo Bühler, C.M. y Naharro, E. (2018). "La Transición" vista desde los valles intermontanos del Noroeste Argentino: nuevos datos de la Quebrada de Los Corrales (El Infiernillo, Tucumán, Argentina). Chungará 50 (1): 71-86.

Orton, C., Tyers, P., Vince, A. (1997) La cerámica en arqueología. Barcelona, España:Crítica. (Orig. 1993).

Pantorrilla Rivas, M. (2009). Análisis de la cerámica Vaquerías del sitio Cabra Corral 8 en Valle de Lerma,
Provincia de Salta. Serie Monográfica y Didáctica 48:144.

Pérez Pieroni, M. J. (2015). Prácticas productivas y tradiciones tecnológicas: la manufactura cerámica prehispánica tardía y colonial en la cuenca sur de pozuelos y el área de santa catalina, puna de jujuy, argentina. Relaciones de la Sociedad Argentina de Antropología 40(1):13-44.

Petrequin, A. M. y Petrequin, P. (1999). La poterie en Novelle Guinée: savoir-faire et trasmision des techiques. Journal de la societé des Océaniestes 108 (1): 71-101.

Pierret, A. (1995) Analyse technologique des cerámiques archaeológiques: developpements méthodologiques pour l'identification des techniques de façonance, un exemple d'application: le matériel du village des Arènes de Levroux (Indre). (Thèse de Doctorat). Paris, France : Universitaires du Septentrion à Villeneuve.

Real Academia Española. (2001). Diccionario de la Lengua Española (DRAE). 22 ${ }^{\text {a }}$ Edición

Rice, P. M. 1987. Pottery Analysis. A Sourcebook. Chicago, USA: The University of Chicago Press.

Roux, V. (1994) La technique du tornage: définition et reconnaissance per les macrotraces. XVe Rencontres Internationales d'Archéologie et d'Historie d'Antibes. Terre Cuite et Société. La céramique, document, technique, economique, culturel, (pp. 45-58).Paris, France: APDCA, Juan-les-Pins

Roux, V. (2019) Ceramics and Society. A Technological Approach to Archaeological Assemblages. Switzerland: Springer Nature.

Rye, 0.1981. Pottery technology. Aldine manuals on Archaeology 4. Washington D.C., USA; Taraxacum.

Stark, M. T.(1999). Social Dimensions of Technical choise in Kalinga Ceramic Traditions. En Chilton E. (ed), Material meanings: critical approaches to the Interpretation of Material Culture (pp 24-43). Salt Lake City, USA: The University of Utah Prees.

Vera, S. D. y De La Fuente, G. A. (2018). Aproximación a los estudios de traza: el caso de las urnas funerarias estilo Saujil (Catamarca, Noroeste Argentino). Anales de Arqueología y Etnología. 73 (2): 145-170.

Visseyrias, A. (2007). Les formes de la tradition: techniques et savoir-faire cerámique à la fin de l'âge du Bronze, entre Rhin et Rhône. Bulletin de la Société préhistorique française 104 (3): 589-609. París.

Wynveldt, F. (2008). Tecnología Cerámica Belén: caracterización macroscópica y conceptualización en la manufactura. Intersecciones en Antropología 8:157-172. 